\title{
Single Center Experience With Hyperthermic Intraperitoneal Chemotherapy
}

\author{
Woo Ram Kim, Hyuk Hur ${ }^{1}$, Byung Soh Min ${ }^{1}$, Seung Hyuk Baik², Kang Young Lee ${ }^{1}$, Nam Kyu Kim ${ }^{1}$ \\ Department of Surgery, CHA Bundang Medical Center, CHA University, Seongnam; ${ }^{1}$ Department of Surgery, Yonsei University College of \\ Medicine, Seoul; ${ }^{2}$ Department of Surgery, Gangnam Severance Hospital, Seoul, Korea
}

Purpose: Cytoreductive surgery (CRS) and hyperthermic intraperitoneal chemotherapy (HIPEC) have been proposed for controlling peritoneal seeding metastasis in some kinds of cancers, including those of colorectal origin, but their safety and oncological benefits are subjects of debate. We present our early experience with those procedures.

Methods: Data were retrospectively collected from all patients with peritoneal carcinomatosis (PC) and pseudomyxoma peritonei (PMP) treated using CRS and HIPEC at Yonsei Cancer Center between July 2014 and July 2015. Short-term outcomes and risk factors for postoperative complications were analyzed.

Results: Twenty-three patients with PC $(\mathrm{n}=18)$ and PMP $(\mathrm{n}=5)$ underwent CRS and HIPEC. Median follow-up and age were 2 months and 54 years, respectively. The median peritoneal carcinomatosis index score was 15, and CC0-1 was achieved in $78.3 \%$ of all patients. The median operation time and bleeding loss were 590 minutes and $570 \mathrm{~mL}$, respectively. Grade-IIIa/grade-IIIb complications occurred in $4.3 \%(n=1) / 26.1 \%(n=6)$ of the patients within 30 days postoperatively, and no 30-day mortalities were reported. Factors related to postoperative complications with CRS and HIPEC were number of organ resection $(\mathrm{P}=0.013)$, longer operation time $(\mathrm{P}<0.001)$, and amount of blood loss $(\mathrm{P}=0.003)$. All patients treated with cetuximab for recurred colorectal cancer had grade-III postoperative complication.

Conclusion: Our initial experience with CRS and HIPEC presented about $30 \%$ grade-III postoperative complications. Therefore, expert surgeons need to perform those procedures with great caution in selected patients who might benefit from it.

\section{Keywords: Cytoreduction Surgical Procedures; Peritoneal metastasis; Colorectal neoplasms; Pseudomyxoma peritonei}

\section{INTRODUCTION}

Peritoneal carcinomatosis (PC) is not an uncommon condition encountered in patients with colorectal cancer and, based on epidemiologic studies, is known to occur in approximately $10 \%-15 \%$ of all patients [1]. The median survival expectancy for patients with PC was reported to be about 7-8 months in untreated patients [2] and 12 months even in patients treated with systemic

Received: August 26, 2016 - Accepted: October 26, 2016

Correspondence to: Nam Kyu Kim, M.D.

Department of Surgery, Yonsei University College of Medicine, Yonsei

University Health System, 50-1 Yonsei-ro, Seodaemun-gu, Seoul 03722, Korea

Tel: +82-2-2228-2117, Fax +82-2-313-8289, E-mail: namkyuk@yuhs.ac

(c) 2017 The Korean Society of Coloproctology

This is an open-access article distributed under the terms of the Creative Commons Attribution NonCommercial License (http://creativecommons.org/licenses/by-nc/4.0) which permits unrestricted non-

commercial use, distribution, and reproduction in any medium, provided the original work is properly cited. chemotherapy [3] until several centers worldwide reported favorable survival outcomes for patients treated using combined cytoreductive surgery (CRS) and hyperthermic intraperitoneal chemotherapy (HIPEC).

Even though the use of a combination of CRS and HIPEC involves considerable risk of complications, as has been reported in several cohort studies and case series $(29 \%-56 \%)$ [4-7], its efficacy in selective patient groups is hardly in dispute. Verwaal [3] reported fascinating survival outcomes in their randomized controlled trial: a median survival of 22.3 months and a significant risk reduction of dying (hazard ratio [HR], 0.55; 95\% confidence interval [CI], 0.32-0.95; log-rank $\mathrm{P}=0.032$ ) in the HIPEC group in comparison to the systemic chemotherapy only group. Moreover, this substantial overall survival is likely to be amplified further when patients to be treated using combined CRS and HIPEC are very carefully selected. Elias et al. [8], one of the leading groups using CRS and HIPEC, reported that patients with isolated, resectable PC could prolong their median survival by up to 
63 months with this aggressive procedure in comparison to a median survival of 24 months with modern chemotherapies. Sugarbaker [9] insisted that a subset of patients with a peritoneal carcinomatosis index (PCI, which quantifies the intraperitoneal tumor burden by distribution and lesion size with a range of 0-39) less than 20 could profit in survival from his experience, and he emphasized the completeness of cytoreduction (CC $0-1)$ for the best survival outcome.

Recently, some leading experts even asserted the necessity for using prophylactic HIPEC. They claimed that early peritoneal metastases are impossible to detect given the absence of symptoms and current limitations of imaging; therefore, proper timing of surgical intervention could be delayed. Indeed, some European centers are performing clinical randomized trials to demonstrate the role of prophylactic HIPEC in selective high-risk subgroups of patients with peritoneal recurrence [10].

In Korea, some centers have been performing combined CRS and HIPEC as an alternative treatment for patients with synchronous or metachronous colorectal cancer with PC since HIPEC was first authorized as a new medical technology by the Ministry of Health and Welfare in late 2013. Even though HIPEC is being widely used for the treatment of PC in Western Europe and the United States, it is still known to involve high morbidity even at centers with experienced surgeons. Furthermore, HIPEC-related short-term outcomes in Korea are not available yet. Therefore, in this paper, we present and discuss our early experience related to HIPEC.

\section{METHODS}

Data were retrospectively collected from all patients with PC and pseudomyxoma peritonei (PMP) treated by using of combination of CRS and HIPEC at Yonsei Cancer Center between July 2014 and July 2015. HIPEC was first introduced and conducted at our institution in July 2014. CRS was performed with intention-totreat in all patients for attaining $\mathrm{R} 0-1$ resections. Data were analyzed to demonstrate the safety of this procedure and the factors related with perioperative adverse events.

\section{Preoperative diagnosis and patient selection}

All the patients referred to our clinic for CRS and HIPEC were thoroughly evaluated in order to exclude possible systemic metastasis other than peritoneal seeding and to determine the tumor burden of PC and PMP. Diagnostic work-ups included esophagogastroduodenoscopy and colonoscopy, as well as computed tomography (CT) scans of the chest, abdomen and pelvis with IV contrast agents. Positron-emission tomography-CT (PET-CT) was considered if extra-abdominal metastasis was suspected or was difficult to determined based on the CT scans.

Patients with synchronous PC originating from colorectal cancer without systemic metastasis were mostly managed by using a combination of CRS and HIPEC, but patients with metachronous
PC, which was incidentally detected by serial elevation of serum carcinoembryonic antigen (CEA) or on a CT scan during the follow-up period after a curative resection, were initially considered as candidates for systemic chemotherapy. The cycles of preoperative chemotherapy were left to the oncologist's decision, but the resectability was discussed by the surgeon and the radiologist every 4-6 cycles of chemotherapy. Also, if the patient requested other treatment options rather than chemotherapy or the oncologist judged continuation of chemotherapy to be impossible because of its toxicity and low patient compliance with the treatment, a multidisciplinary team approach was recommended to the patient, the treatment plan was discussed further, and finally a decision was made to perform CRS and HIPEC when the tumor was such that a complete resection was thought to be possible, provided that the patient would benefit from the procedure. At out institution, PMP with obstructive symptoms or impending obstruction was considered to be an indication for the use of combined CRS and HIPEC.

\section{Determination of CRS and HIPEC}

Preoperative diagnostic laparoscopy was not routinely performed for the evaluation of resectability. Its use was entirely left to the surgeon. Mostly, the way to proceed for the CRS and HIPEC was decided under direct vision after a long midline abdominal incision had been made from the xiphoid process to the symphysis pubis. The extent of PC was determined at the time of initial surgical exploration by using the PCI score. The PCI score, which was first suggested for the staging of PC by Jacquet and Sugarbaker [11] in 1996 and which comprises 13 abdominopelvic regions with lesion size scores and is a summed numerical score from 0 to 39, was adopted at our institution to investigate the extent of PC and to help surgeons determine how to apply the procedure. However, the indications for the use of CRS and HIPEC at our institution do not coincide with Sugarbaker [9]'s decision algorithm, but rather are based on those agreed on at a 2006 HIPEC consensus meeting in Milano, Italy [12]. In other words, a combination of CRS and HIPEC is used when complete cytoreduction is possible, regardless of the PCI score.

\section{CRS and HIPEC procedure}

CRS included removing the primary tumor with acceptable resection margins, metastatic lymph nodes, involved organs, and all peritoneal seeding metastases in the abdominopelvic cavity. If the tumors were unresectable because of anatomical location, including mesenteries of the small and the large bowels, a high-voltage monopolar device was used for cauterization of multiple small lesions. A peritonectomy was not conducted in any of the patients; rather, a peritonectomy was performed if visual peritoneal seeding metastases were confirmed at the initial surgical exploration. Before HIPEC was started, the completeness of cytoreduction (CC score from 0 to 3) [9] was measured and recorded.

HIPEC was facilitated using the open (coliseum) technique, 
which was prepared by suturing 1-0 vicryl to the deep layer of the epidermis and subcutaneous area and fixing it to an Omni retractor with mosquito instruments. Initially, $3 \mathrm{~L}$ of heated perfusion solution was infused into the abdominal cavity at a rate of 600$800 \mathrm{~mL} / \mathrm{min}$ through the inflow tube by way of a Belmont hyperthermic pump. When the temperature of the abdominal cavity had reached $40^{\circ} \mathrm{C}-41^{\circ} \mathrm{C}$, the first dose of mitomycin-C of 17.5 $\mathrm{mg} / \mathrm{m}^{2}$ was mixed into the heated solution, after which a dose of $8.8 \mathrm{mg} / \mathrm{m}^{2}$ was added every 30 minutes. The temperature of the perfusion solution was maintained and evenly distributed at $41^{\circ} \mathrm{C}-42^{\circ} \mathrm{C}$ by stirring with a surgeon's hand. The duration of the HIPEC procedure was 90 minutes, after which the perfusion solution was completely drained and a bowel anastomosis was performed, if needed. All patients were taken to the intensive care unit for short-term observation.

\section{Postoperative chemotherapy and follow-up}

Adjuvant chemotherapeutic regimens were mainly with FOLFOX (oxaliplatin, leucovorin and 5-FU) or FOLFIRI (irinotecan, leu- covorin and 5-FU) with or without biologic agents. Postoperative chemotherapy was recommended for all patients after they had completely recovered from surgical stress without any complication.

After the completion of postoperative chemotherapy, clinical evaluations were performed regularly every 3 months for the first 2 years and thereafter every 6 months for the subsequent 3 years. Serum CEA was measured whenever the patient visited the outpatient clinic, and CT scans of the chest, abdomen and pelvis were performed every 6 months during the follow-up period.

\section{Statistical analysis}

Patients were grouped according to the Clavien-Dindo classification [13]. Group A, which was grades 0-II, included patients with mild complications without any need for further intervention, and group B, which was more than grade III, included patients with moderate-to-severe complication with need for surgical exploration or radiological/medical intervention. Each factor associated with a postoperative complication after CRS and HIPEC

Table 1. Case summary of CRS + HIPEC

\begin{tabular}{|c|c|c|c|c|c|c|}
\hline Type & No. & Sex/age (yr) & Origin & $\mathrm{PCl}$ & CC & Complication \\
\hline \multirow[t]{18}{*}{ PC } & 1 & $\mathrm{M} / 48$ & Ascending colon cancer & 20 & 0 & Gastric stasis \\
\hline & 2 & $\mathrm{~F} / 47$ & Rectal cancer & 22 & 1 & Wound seroma \\
\hline & 3 & $M / 64$ & Cecal cancer & 9 & 0 & - \\
\hline & 4 & $F / 48$ & Ascending colon cancer & 15 & 1 & Wound seroma \\
\hline & 5 & $\mathrm{~F} / 47$ & Transverse colon cancer & 16 & 1 & - \\
\hline & 6 & $\mathrm{M} / 72$ & Ascending colon cancer & 3 & 0 & - \\
\hline & 7 & $M / 37$ & Appendiceal cancer & 9 & 0 & - \\
\hline & 8 & $F / 54$ & Ovarian cancer & 12 & 0 & Pelvic abscess \\
\hline & 9 & $\mathrm{M} / 43$ & Appendiceal cancer & 28 & 2 & - \\
\hline & 10 & M/39 & Sigmoid colon cancer & 2 & 0 & Urinary retention \\
\hline & 11 & M/66 & Ascending colon cancer & 12 & 1 & Intestinal obstruction \\
\hline & 12 & $M / 64$ & Cecal cancer & 16 & 1 & Postoperative bleeding \\
\hline & 13 & $F / 54$ & Ovarian cancer & 6 & 0 & - \\
\hline & 14 & $\mathrm{M} / 63$ & Sigmoid colon cancer & 4 & 0 & - \\
\hline & 15 & $\mathrm{M} / 62$ & Ascending colon cancer & 10 & 1 & Pleural effusion \\
\hline & 16 & $\mathrm{~F} / 50$ & Ascending colon cancer & 30 & 2 & - \\
\hline & 17 & M/69 & Cecal cancer & 13 & 1 & - \\
\hline & 18 & $F / 36$ & Sigmoid colon cancer & 17 & 1 & - \\
\hline \multirow[t]{5}{*}{ PMP } & 1 & $\mathrm{~F} / 61$ & Appendiceal cancer & 39 & 2 & Intestinal obstruction \\
\hline & 2 & $\mathrm{M} / 60$ & Appendiceal cancer & 24 & 2 & Intestinal obstruction \\
\hline & 3 & $\mathrm{M} / 67$ & Ascending colon cancer & 13 & 1 & Pleural effusion \\
\hline & 4 & M/63 & Sigmoid colon cancer & 28 & 2 & Intra-abdominal abscess/lleus \\
\hline & 5 & $\mathrm{M} / 43$ & Appendiceal cancer & 39 & 1 & Anastomotic leakage \\
\hline
\end{tabular}

CRS + HIPEC, cytoreductive surgery + hyperthermic intraperitoneal chemotherapy; PC, peritoneal carcinomatosis; PMP, pseudomyxoma peritonei; PCl, peritoneal carcinomatosis index; CC, completeness of cytoreduction. 
was divided into 2 groups by using the cutoff value from the receiver operating characteristic curve and was analyzed using the chi-square test. All P-values were two-sided, and $\mathrm{P}<0.05$ was considered statistically significant. All statistical analyses were carried out using the IBM SPSS Statistics ver. 20.0 (IBM Co., Armonk, NY, USA).

\section{RESULTS}

A total of 23 patients with PC $(n=18)$ and PMP $(n=5)$ were considered for CRS and HIPEC with intention-to-treat (Table 1). The median follow-up period was 2 months (range, 0-11 months), and the median age was 54 years old (range, 36-72 years). Among these patients, $39.1 \%(n=9)$ had an American Society of Anaesthesiologists physical status classification grade of more than III, and $13 \%(n=3)$ were moderate-to-severe malnutrition status. All the patients with synchronous PC had a CRS

Table 2. Patient's characteristics $(n=23)$

\begin{tabular}{|c|c|}
\hline Variable & Value \\
\hline \multicolumn{2}{|l|}{ Sex } \\
\hline Male & $15(65.2)$ \\
\hline Female & $8(34.8)$ \\
\hline Age (yr) & $54(36-72)$ \\
\hline \multicolumn{2}{|l|}{ Preoperative PNI ${ }^{a}$} \\
\hline Normal-mild ( $\geq 40)$ & $20(87.0)$ \\
\hline Moderate (35-40) & $1(4.3)$ \\
\hline Severe $(<35)$ & $2(8.7)$ \\
\hline Preoperative CEA level (ng/ $\mu \mathrm{L})$ & $13.0(2.0-141.0)$ \\
\hline \multicolumn{2}{|l|}{ ASA physical status classification } \\
\hline $1-\|$ & $14(60.9)$ \\
\hline$\geq \mid 11$ & $9(39.1)$ \\
\hline \multicolumn{2}{|l|}{ PC timing } \\
\hline Synchronous & $7(30.4)$ \\
\hline Metachronous & $16(69.6)$ \\
\hline Time interval since last surgery (mo) & $24(5-52)$ \\
\hline \multicolumn{2}{|l|}{ Neoadjuvant chemotherapy } \\
\hline Yes & $16(69.6)$ \\
\hline No & $7(30.4)$ \\
\hline Neoadjuvant chemotherapy cycles & $12(0-93)$ \\
\hline \multicolumn{2}{|l|}{ Adjuvant chemotherapy } \\
\hline Yes & $11(47.8)$ \\
\hline No & $12(52.1)$ \\
\hline
\end{tabular}

Values are presented as number (\%) or median (range).

CEA, carcinoembryonic antigen; ASA, American Society of Anaesthesiologists; PC, peritoneal carcinomatosis.

aPrognostic nutrional index (normal, $\geq 50$; mild, 40-50; moderate, 35-40; severe, $<35)$. and HIPEC without any neoadjuvant chemotherapy. However, patients with metachronous PC mostly had undergone preoperative chemotherapy, a median of 12 cycles (range, 0-93 cycles) for 24 months (range, 5-52 months), during the time from their last surgery until the CRS and HIPEC was performed (Table 2).

The primary tumor origin of PC in patients with colorectal cancer was mostly the right-side colon $(75 \%, 12$ out of 16$)$. The median PCI score was 15 (range, 2-39), and patients with PCI scores of more than 20 were included in this study $(n=7,30.4 \%)$. Complete cytoreduction was possible in $78.3 \%(n=18)$ of the patients, but in $21.7 \%(\mathrm{n}=5)$ of the patients, the resection was an $\mathrm{R} 2$ resection. The median operation time and the bleeding loss were 590 minutes (range, 350-1,225 minutes) and $570 \mathrm{~mL}$ (range, 80-3,250 $\mathrm{mL}$ ), respectively, and 9 patients $(39.1 \%)$ required a transfusion during operation (Table 3).

Most patients recovered and were discharged a median of 19 days (range, 8-101 days) after surgery. Adverse events during the first 30 days following surgery occurred in 12 (12 of 23, 52.2\%) patients, and among those 12, 7 (7 of 12, 58.3\%) needed further radiological intervention or surgical exploration. Moreover, late complications occurred in 2 of the 23 patients (8.7\%), for whom delayed surgical intervention was needed. Therefore, overall 9 of the 23 patients (39.1\%) who underwent CRS and HIPEC had more than grade-III complications according to the ClavienDindo classification [13]. No mortalities occurred during the first 30 days of the follow-up period, but disease-related deaths occurred in 3 patients (13.0\%) (Table 4 ).

Factors such as organs resected (more than 3), longer operation time ( $\geq 630$ minutes), and large amount of blood loss $(\geq 600 \mathrm{~mL})$ during surgery were found to be associated with a high rate of postoperative complications. Interestingly, all 4 patients with recurred colorectal cancer who had been treated with cetuximab had grade-III postoperative complications. However, anastomotic leakage occurred in only 1 patient, who had a complete total colectomy with ileorectal anastomosis and HIPEC because of PMP. Preoperative nutritional status and number of bowel anastomoses were not closely related to the occurrence of postoperative adverse events (Table 5).

\section{DISCUSSION}

Before HIPEC was permitted for the treatment of patients with colorectal cancer with PC in Korea, the only option for patients with PC was systemic chemotherapy with or without biologic agents. Even though newly developed chemotherapeutic and biologic agents have prolonged the life expectancy in those patients [14], chemotherapy-related adverse events or intolerance and resistance to this therapy ultimately made it impossible to prevent tumor progression and led to withdrawal of the treatment.

Our institution initially had difficulty in enrolling patients for CRS and HIPEC because our oncologists and gastroenterologists had some suspicions about this new procedure with regards to its 
Table 3. Tumor characteristics and intraoperative parameters

\begin{tabular}{|c|c|}
\hline Variable & Value \\
\hline \multicolumn{2}{|l|}{ Primary tumor } \\
\hline Appendix & 5 \\
\hline Cecum & 3 \\
\hline Ascending colon & 7 \\
\hline Transverse colon & 1 \\
\hline Sigmoid colon & 4 \\
\hline Rectum & 1 \\
\hline Others & 2 \\
\hline \multicolumn{2}{|l|}{ Histopathology } \\
\hline Adenocarcinoma, well/moderately differentiated & 12 \\
\hline Adenocarcinoma, poorly differentiated/mucinous & 9 \\
\hline Serous cystadenocarcinoma (ovary origin) & 2 \\
\hline \multicolumn{2}{|l|}{ Recurred tumor } \\
\hline PC & $18(78.3)$ \\
\hline Right-sided colon cancer & 12 \\
\hline Left-sided colon cancer & 4 \\
\hline Ovarian cancer & 2 \\
\hline PMP & $5(21.7)$ \\
\hline Appendix origin & 3 \\
\hline Ascending colon & 1 \\
\hline Sigmoid colon & 1 \\
\hline \multicolumn{2}{|l|}{$\mathrm{PCl}$ score } \\
\hline$\leq 20$ & $16(69.6)$ \\
\hline$>20$ & $7(30.4)$ \\
\hline PCl score & $15(2-39)$ \\
\hline \multicolumn{2}{|l|}{ CC score $(n, \%)$} \\
\hline $0-1$ & $18(78.3)$ \\
\hline $2-3$ & $5(21.7)$ \\
\hline \multicolumn{2}{|l|}{ Completeness of resection } \\
\hline Ro & $9(39.1)$ \\
\hline $\mathrm{R} 1$ & $9(39.1)$ \\
\hline $\mathrm{R} 2$ & $5(21.7)$ \\
\hline Operation time (hr) & $590(350-1,225)$ \\
\hline Bleeding loss (mL) & $570(80-3,250)$ \\
\hline \multicolumn{2}{|l|}{ Transfusion } \\
\hline Yes & $9(39.1)$ \\
\hline No & $14(60.9)$ \\
\hline
\end{tabular}

Values are presented as number (\%) or median (range).

$\mathrm{PC}$, peritoneal carcinomatosis; PMP, pseudomyxoma peritonei; $\mathrm{PCl}$, peirtoneal carcinomatosis index; CC, completeness of cytoreduction.

efficacy and safety. This distrust made them hesitate to introduce this new therapeutic option to their patients in whom the disease
Table 4. Short-term outcomes after CRS + HIPEC $(n=23)$

\begin{tabular}{lc}
\hline Variable & Value \\
\hline Follow-up (mo) & $2(0-11)$ \\
Hospital stay (day) & $19(8-101)$ \\
30 Days - AE & $12(52.2)$ \\
Grades I-II & $5(21.7)$ \\
Grade IIla & $1(4.3)$ \\
$\geq$ Grade IIlb & $6(26.1)$ \\
Postoperative IPC-related neutropenia & $3(13.0)$ \\
Mild & 2 \\
Moderate & 1 \\
Severe & 0 \\
Late AE & $2(8.7)$ \\
Enterovaginal fistula & 1 \\
\hline Intestinal obstruction & 1 \\
\hline 30 Days - mortality & 0 \\
Postoperative disease status & \\
No recurrence or stable disease & $18(65.2)$ \\
Recurrence or progression of disease & $5(34.8)$ \\
Death during follow-up & $3(13.0)$ \\
Disease related & 3 \\
Disease unrelated & 0 \\
\hline
\end{tabular}

Values are presented as median (range) or number (\%).

CRS + HIPEC, cytoreductive surgery + hyperthermic intraperitoneal chemotherapy; IPC, intraperitoneal chemotherapy.

${ }^{\mathrm{a}} \mathrm{AE}$, adverse event according to the Clavien-Dindo classification. 'ate AE: complications 30 days after CRS + HIPEC.

had been stabilized with continuous chemotherapy. The high complication rates previously reported in many centers worldwide [4-7] and the existence of only a few randomized controlled trials were the main obstacles to persuading them to enroll their patients who were suffering from metachronous PC. Therefore, the first enrolled patient was one who had colorectal cancer with synchronous PC and who had been directly referred to our department by a local clinic. This patient was initially diagnosed with ascending colon cancer with a few peritoneal seeding nodules based on the CT scan (PCI score, 8), but the intraoperative PCI score eventually revealed more than what had been expected preoperatively (intraoperative PCI score, 20). Even though this patient temporarily had gastric stasis, he recovered completely three weeks later and received adjuvant chemotherapy afterwards. He has shown no evidence of tumor recurrence since then. After the first patient had recovered without any major complication and HIPEC had been shown not to delay adjuvant chemotherapy, patients with metachronous PC started to be enrolled in HIPEC therapy by oncologists and gastroenterologists. As Tables 1 and 2 show, not only patients with PC $(\mathrm{n}=2)$ originating from other 
Table 5. Factors associated with postoperative complications after CRS + HIPEC

\begin{tabular}{|c|c|c|c|}
\hline Variable & $\begin{array}{l}\text { Group A } \\
(n=14)\end{array}$ & $\begin{array}{l}\text { Group B } \\
(\mathrm{n}=9)\end{array}$ & P-value \\
\hline $\mathrm{Age}^{\mathrm{a}}$ & & & 0.214 \\
\hline$<55$ & $9(64.3)$ & $3(33.3)$ & \\
\hline$\geq 55$ & $5(35.7)$ & $6(66.7)$ & \\
\hline Body mass index $\left(\mathrm{kg} / \mathrm{m}^{2}\right)$ & & & 0.197 \\
\hline$<21$ & $6(42.9)$ & $7(77.8)$ & \\
\hline$\geq 21$ & $8(57.1)$ & $2(22.2)$ & \\
\hline No. of organs resected & & & 0.013 \\
\hline $0-2$ & 11 (78.6) & $2(22.2)$ & \\
\hline$\geq 3$ & $3(21.4)$ & $7(77.8)$ & \\
\hline No. of bowel anastomosis & & & 0.391 \\
\hline $0-2$ & $14(100)$ & $8(88.9)$ & \\
\hline$\geq 3$ & $0(0)$ & $1(11.1)$ & \\
\hline Previous operation & & & 0.176 \\
\hline No & $6(42.9)$ & $1(11.1)$ & \\
\hline Yes & $8(57.1)$ & $8(88.9)$ & \\
\hline PCl index ${ }^{a}$ & & & 0.214 \\
\hline$<15$ & $9(64.3)$ & $3(33.3)$ & \\
\hline$\geq 15$ & $5(35.7)$ & $6(66.7)$ & \\
\hline ASA score & & & 0.999 \\
\hline $1-2$ & $9(64.3)$ & $5(55.6)$ & \\
\hline$\geq 3$ & $5(35.7)$ & $4(44.4)$ & \\
\hline Operation time (min) ${ }^{\mathrm{a}}$ & & & $<0.001$ \\
\hline$<630$ & $12(85.7)$ & $0(0)$ & \\
\hline$\geq 630$ & $2(14.3)$ & $9(100)$ & \\
\hline Blood loss (mL) ${ }^{\mathrm{a}}$ & & & 0.003 \\
\hline$<600$ & $11(78.6)$ & $1(11.1)$ & \\
\hline$\geq 600$ & $3(21.4)$ & $8(88.9)$ & \\
\hline Transfusion & & & 0.077 \\
\hline No & $11(78.6)$ & $3(33.3)$ & \\
\hline Yes & $3(21.4)$ & $6(66.7)$ & \\
\hline Cycles of preoperative chemotherapy ${ }^{\mathrm{a}}$ & & & 0.383 \\
\hline$<17$ & $10(71.4)$ & $4(44.4)$ & \\
\hline$\geq 17$ & $4(28.6)$ & $5(55.6)$ & \\
\hline Preoperative biologic agent & & & 0.017 \\
\hline None & $11(78.6)$ & $5(55.6)$ & \\
\hline Cetuximab & $0(0)$ & $4(44.4)$ & \\
\hline Avastin & $2(14.3)$ & $0(0)$ & \\
\hline Avastin + cetuximab & $1(7.1)$ & $0(0)$ & \\
\hline Preoperative PNI ${ }^{b}$ & & & 0.538 \\
\hline Normal-mild & 13 (92.9) & $7(77.8)$ & \\
\hline Moderate-severe & $1(7.1)$ & $2(22.2)$ & \\
\hline
\end{tabular}

CRS + HIPEC, cytoreductive surgery + hyperthermic intraperitoneal chemotherapy. Group A, Clavien-Dindo classification grades 0-\|l; group B, Clavien-Dindo classification grade $\geq$ III.

${ }^{a}$ Cutoff value obtained from the receiver operating characteristic curve. ${ }^{\text {bPrognos- }}$ tic nutrional index (normal, $\geq 50$; mild, 40-50; moderate, 35-40; severe, $<35$ ). sites such as the ovaries but also patients with PMP $(n=5)$ were included in the analysis because the main purpose of our study was to introduce our early experiences with respect to the safety of this new procedure in a single center.

As for HIPEC-associated complications, 3 adverse events (3 of 7, $14.3 \%$ ), urinary retention, gastric stasis, and intestinal obstruction, occurred in patients with synchronous PC, and the patient with intestinal obstruction was eventually treated with surgical intervention. To be frank, this patient was the first and the last patient enrolled in HIPEC despite his having a nonprogressive single hepatic metastasis after completion of 12 cycles of neoadjuvant chemotherapy, which is known to be a contraindication for HIPEC. During a 660-minute surgery, CRS and intraoperative radio-frequency ablation to a single hepatic metastasis were performed, attaining a R0 resection. Among 16 patients with metachronous PC or PMP, 9 patients (56.3\%) had postoperative complications within 30 days. Excluding 3 patients with minor adverse events, 6 other patients with major complications (37.5\%), such as intra-abdominal abscess, pleural effusion, intestinal obstruction, immediate postoperative bleeding and anastomotic leakage, were finally treated with radiologic or surgical interventions.

Given our short-term outcomes, the major complication rate for patients with synchronous PC tended to be lower than it was for patients with metachronous PC. This is the reason patients with metachronous PC commonly present with conditions that are unfavorable for surgery, including severe adhesion, undernutritional status and previous multiple cycles of chemotherapy with or without biologic agents. As Table 5 shows, all 4 patients treated with cetuximab preoperatively had major complications, such as pleural effusion, intestinal obstruction, immediate postoperative bleeding and intra-abdominal abscess with sepsis. In a recent European study, bevacizumab was also found to be associated with a twofold increased morbidity in patients treated with CRS and HIPEC (odds ratio, 2.28; 95\% CI, 1.05 to 4.95; $\mathrm{P}=0.04$ ) [15]. Therefore, great attention is needed when performing CRS and HIPEC, particularly in the patients who have metachronous PC or have received preoperative chemotherapy or biologic therapy.

In addition to the aforementioned preoperative unfavorable conditions, the major complications tended to be associated with several intraoperative factors, as shown in Table 5. Major complications occurred more commonly in patients who had more than 3 organs resected, operation times of 630 minutes or longer, and losses of $600 \mathrm{~mL}$ or more of blood during surgery. Against our expectation, anastomotic leakage occurred in only one patient (4.3\%) and showed no significant differences compared to usual colorectal surgery. A great diversity of opinion exists concerning this early postoperative major complication rate ( 7 of $23,30.4 \%$ ), i.e., whether it needs to be abided for better oncologic outcomes or not. However, if we can attain the same oncologic outcomes as leading experts in Europe and the United States do, we think this aggressive procedure, with its accompanying high morbidity, should be considered for carefully selected patients who might 
benefit from it use.

In conclusion, our initial experience with CRS and HIPEC showed that about $30 \%$ of the patients experienced grade-III postoperative complications, which was strongly associated with the number of organs resected, longer operation time, and the amount of blood loss. Therefore, an expert surgeon needs to perform this combined procedure with great caution and only on selected patients who might benefit from it.

\section{CONFLICT OF INTEREST}

No potential conflict of interest relevant to this article was reported.

\section{REFERENCES}

1. Jayne DG, Fook S, Loi C, Seow-Choen F. Peritoneal carcinomatosis from colorectal cancer. Br J Surg 2002;89:1545-50.

2. Lemmens VE, Klaver YL, Verwaal VJ, Rutten HJ, Coebergh JW, de Hingh IH. Predictors and survival of synchronous peritoneal carcinomatosis of colorectal origin: a population-based study. Int J Cancer 2011;128:2717-25.

3. Verwaal VJ. Long-term results of cytoreduction and HIPEC followed by systemic chemotherapy. Cancer J 2009;15:212-5.

4. van Leeuwen BL, Graf W, Pahlman L, Mahteme H. Swedish experience with peritonectomy and HIPEC. HIPEC in peritoneal carcinomatosis. Ann Surg Oncol 2008;15:745-53.

5. Cashin PH, Graf W, Nygren P, Mahteme H. Cytoreductive surgery and intraperitoneal chemotherapy for colorectal peritoneal carcinomatosis: prognosis and treatment of recurrences in a cohort study. Eur J Surg Oncol 2012;38:509-15.

6. Verwaal VJ, van Ruth S, de Bree E, van Sloothen GW, van Tinteren $\mathrm{H}$, Boot $\mathrm{H}$, et al. Randomized trial of cytoreduction and hyperthermic intraperitoneal chemotherapy versus systemic chemotherapy and palliative surgery in patients with peritoneal carcinomatosis of colorectal cancer. J Clin Oncol 2003;21:3737-43.
7. Huang CQ, Feng JP, Yang XJ, Li Y. Cytoreductive surgery plus hyperthermic intraperitoneal chemotherapy improves survival of patients with peritoneal carcinomatosis from colorectal cancer: a case-control study from a Chinese center. J Surg Oncol 2014;109: 730-9.

8. Elias D, Lefevre JH, Chevalier J, Brouquet A, Marchal F, Classe $\mathrm{JM}$, et al. Complete cytoreductive surgery plus intraperitoneal chemohyperthermia with oxaliplatin for peritoneal carcinomatosis of colorectal origin. J Clin Oncol 2009;27:681-5.

9. Sugarbaker PH. Successful management of microscopic residual disease in large bowel cancer. Cancer Chemother Pharmacol 1999; 43 Suppl:S15-25.

10. Baratti D, Kusamura S, Deraco M. Prevention and early treatment of peritoneal metastases from colorectal cancer: second-look laparotomy or prophylactic HIPEC? J Surg Oncol 2014;109:225-6.

11. Jacquet P, Sugarbaker PH. Clinical research methodologies in diagnosis and staging of patients with peritoneal carcinomatosis. Cancer Treat Res 1996;82:359-74.

12. Esquivel J, Sticca R, Sugarbaker P, Levine E, Yan TD, Alexander R, et al. Cytoreductive surgery and hyperthermic intraperitoneal chemotherapy in the management of peritoneal surface malignancies of colonic origin: a consensus statement. Society of Surgical Oncology. Ann Surg Oncol 2007;14:128-33.

13. Dindo D, Demartines N, Clavien PA. Classification of surgical complications: a new proposal with evaluation in a cohort of 6336 patients and results of a survey. Ann Surg 2004;240:205-13.

14. Klaver YL, Leenders BJ, Creemers GJ, Rutten HJ, Verwaal VJ, Lemmens VE, et al. Addition of biological therapies to palliative chemotherapy prolongs survival in patients with peritoneal carcinomatosis of colorectal origin. Am J Clin Oncol 2013;36:157-61.

15. Eveno C, Passot G, Goéré D, Soyer P, Gayat E, Glehen O, et al. Bevacizumab doubles the early postoperative complication rate after cytoreductive surgery with hyperthermic intraperitoneal chemotherapy (HIPEC) for peritoneal carcinomatosis of colorectal origin. Ann Surg Oncol 2014;21:1792-800. 